\title{
Artefact
}

Techniques, histoire et sciences humaines

$11 \mid 2019$

Pannes et accidents (XIX $\mathrm{X}^{\mathrm{e}} \mathrm{XX|^{ \textrm {e } }}$ siècle)

\section{Marie Bouhaïk-Girones, Olivier Spina et Mélanie Traversier (dir.), « Mécanique de la représentation : machines et effets spéciaux sur les scènes européennes, $\mathrm{XV}^{\mathrm{e}}-\mathrm{XVIII}{ }^{\mathrm{e}}$ siècles »}

Numéro spécial, Revue d'Histoire du Théâtre, n² 278, 2018

\section{Marie Thébaud-Sorger}

\section{OpenEdition \\ Journals}

Édition électronique

URL : http://journals.openedition.org/artefact/5103

DOI : 10.4000/artefact.5103

ISSN : 2606-9245

Éditeur :

Association Artefact. Techniques histoire et sciences humaines, Presses universitaires du Midi

Édition imprimée

Date de publication : 15 décembre 2019

Pagination : $344-350$

ISBN : 978-2-8107-0667-9

ISSN : 2273-0753

Référence électronique

Marie Thébaud-Sorger, « Marie Bouhaïk-Girones, Olivier Spina et Mélanie Traversier (dir.), « Mécanique de la représentation : machines et effets spéciaux sur les scènes européennes, xve-xvIII siècles » », Artefact [En ligne], 11 | 2019, mis en ligne le 27 novembre 2020, consulté le 30 novembre 2020. URL: http://journals.openedition.org/artefact/5103; DOI : https://doi.org/10.4000/artefact.5103

Ce document a été généré automatiquement le 30 novembre 2020.

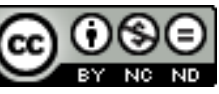

Artefact, Techniques, histoire et sciences humaines est mise à disposition selon les termes de la Licence Creative Commons Attribution - Pas d'Utilisation Commerciale - Pas de Modification 4.0 International. 
Marie Bouhailk-Girones, Olivier Spina et Mélanie Traversier (dir.), "Mécanique de la représentation: machines et effets spéciaux sur les scènes européennes, $\mathrm{XV}^{\mathrm{e}}$ XVIII ${ }^{\mathrm{e}}$ siècles »

Numéro spécial, Revue d'Histoire du Théâtre, nº 278, 2018

Marie Thébaud-Sorger

\section{RÉFÉRENCE}

Marie Bouhaïk-Girones, Olivier Spina et Mélanie Traversier (dir.), « Mécanique de la représentation : machines et effets spéciaux sur les scènes européennes, $\mathrm{XV}^{\mathrm{e}}$ XVIII siècles ", Numéro spécial, Revue d'Histoire du Théâtre, n 278, 2018, 160 p.

1 Le petit numéro spécial se compose de neufarticles très riches et d'une belle introduction de Mélanie Traversier qui dresse le cadre des techniques et techniciens du spectaculaire aux $\mathrm{XV}^{\mathrm{e}}$-XVIII ${ }^{\mathrm{e}}$ siècle. La diversité des cas étudiés se déploie sur une large période chronologique, depuis les mystères $d u x^{e}-x v I^{e}$ siècle en France (Marie Bouhaïk-Girones) jusqu'au renouvellement scénique porté par l'ébullition de la scène italienne à la Renaissance (Paola Ventrone sur les scènes florentines $\mathrm{du}_{\mathrm{Xv}} \mathrm{e}$ siècle et Pascal Brioist sur les machines de spectacles de Léonard de Vinci), la circulation européenne de ces savoirs scéniques transitant par les spectacles au service des cours et des Princes, entre la cour de France et de Lorraine (Vincent Dorothée), en lien avec les scènes publiques, qu'il s'agisse du théâtre baroque parisien $\mathrm{du} \mathrm{XVI}^{\mathrm{e}}$-XvII ${ }^{\mathrm{e}}$ siècle (Anne Surgers) ou de la scène élisabéthaine (Olivier Spina), enfin les mutations de genre et 
d'esthétique sur des scènes plus institutionnalisées au XvIII ${ }^{\mathrm{e}}$ siècle en France comme le ballet à l'Académie royale de musique (Barbara Nestola) et les traductions de la machinerie d'Opéra dans l'Encyclopédie (Laura Naudeix). Des représentations à vocation civique ou religieuse, au service du prestige princier (entrées de villes, fêtes royales et théâtres de cour) jusqu'aux divertissements publics, cet ensemble de configurations variées permet de voir en situation comment les décors, effets spéciaux et machineries mis au service de la représentation traduisent des usages différenciés de l'ingéniosité technique en reformulant les problématiques habituelles liées aux questions de genre et d'esthétique. Le numéro participe donc de ce fait du renouvellement porté par l'histoire des techniques qui, inversement, bénéficie de cet éclairage sur la place du théâtre comme espace central dans la formation et la circulation des savoirs techniques et mécaniques à l'époque moderne ${ }^{1}$. La cohérence forte du propos d'ensemble du numéro s'articule à différents niveaux. Chaque article met en exergue des corpus inédits d'archives, cahiers de secrets, registres comptables, quittances, commandes, contrats, dessins, qui permettent de redonner corps à la matérialité de la présentation, de la scène et surtout des métiers impliqués dans l'élaboration des spectacles: maître-feinteurs, artificiers ingénieurs, artisans, machiniste-scénographes. À partir de la variété des dispositifs techniques (poulies, escamotage, systèmes de mise à feu, etc.) et des machines imaginées pour produire des effets, c'est une réflexion d'ensemble qui peut s'esquisser : d'une part, sur la place et les mutations de l'esthétique du merveilleux dans la culture de l'Europe moderne; et d'autre part, sur la place de la technique et celle des machines comme outil conceptuel et expérimental pour penser "l'ingéniosité des artifices et augmentation les forces humaines" - pour reprendre la définition de Furetière (rappelée par Vincent Dorothée) - à la croisée des savoirs conceptuels de la mécanique et savoir faire de métiers.

2 Les articles ainsi font dialoguer des sources imprimées, traités, planches éditées avec de nombreux manuscrits qui détaillent les éléments fournis, éléments de décors, machineries, systèmes d'escamotage, accessoires, toiles peintes etc. mais aussi la nature du travail accompli et les matériaux utilisés. La fabrication mobilise des savoir-faire que l'on peut pister dans les contrats et baux passés avec les autorités, les villes et les souverains. Dès le $\mathrm{xv}^{\mathrm{e}}$ siècle, on peut distinguer deux types de machines de théâtre : d'une part, les dispositifs scénographiques, décors cachés, système de poulies, poids et engrenages, et d'autre part, les automates qui se mouvaient sur scène, oiseaux mécaniques, chariots automobiles, etc. Les effets spéciaux (lumineux, olfactifs, sonores, comme le tonnerre) constituent une catégorie bien identifiée requérant des compétences spécifiques : la maîtrise du feu par exemple est une des spécialités de ces " maîtres de secrets ", peintre-feintiers qui fournissent pour les mystères une fournaise " miracle», ou un "diable couvert de feu ». Les artificiers s'imposent ensuite comme une figure hybride entre artisan, ingénieur-scénographe et décorateur feinteur, à l'instar de Tommaso Francini (1571-1661) ou d'Horace Morel (v. 1568-1636) pour la cour de Navarre et de Lorraine. Dépassant sa fonction de « commissaire général des feux et artifice de France ", il est plus un véritable imprésario, faisant office de régisseurmachiniste voire d'ordonnateur pour les ballets lorrains (p. 87) en étendant son activité à celle des machines (le ballet des fées en 1625). La polyvalence est bien la première caractéristique de ces maîtres d'œuvre de talent, et n'est pas réductible aux scénographes liés aux ingénieurs italiens. John Carow, par exemple, un artisan londonien travaillant pour les scènes élisabéthaines et particulièrement pour l'office 
des divertissements de la maison royale pendant plus de vingt ans (1552-1575) est décrit comme ébéniste; mais c'est un maître d'œuvre qui coordonne en réalité le travail de nombreux artisans, experts, apprentis et compagnons. L'attention à ces corpus permet également de mieux détailler le monde des métiers de la représentation, machinistes, habilleurs, dessinateurs qui entourent et accompagnent les productions.

Par ailleurs, prendre en compte cette polyvalence permet de réévaluer les dichotomies habituelles entre les scènes et de mieux sérier la circulation des savoirs techniques entre les milieux de spécialistes et les aires géographiques. Certes le service pour les cours a constitué une matrice essentielle pour capitaliser et mettre en œuvre la magnificence des artifices et déployer tout un arsenal de savoir-faire : maîtrise du feu, savoir de pointe de la mécanique, automates étonnants... Vinci se forme à l'atelier de Verrocchio qui supervise l'entrée triomphale du duc de Sforza à Milan, puis accompagne la cour de Charles d'Amboise, concepteur des fêtes d'Amboise et Romorantin pour François $\mathrm{I}^{\mathrm{er}}$ (noces, commémoration de Marignan). Ce sont aussi des ingénieurs et scénographes, officiant dans les jardins princiers autant que sur les scènes, comme l'ingénieur hydraulique Tommaso Francini, auteur de jeux d'eau et d'automates pour les jardins de Pratolino qui devient intendant général des eaux et fontaines de France en 1623. Horace Morel quant à lui officie également sur les scènes publiques parisiennes: théâtre du jeu de paume, du Marais, hôtel de Bourgogne. L'analyse des techniques révèle également, comme le montre Olivier Spina, que la diversité des scènes élisabéthaines, théâtres élitaires privés ou de cour et amphithéâtres populaires, demeure unifiée du fait de ce même milieu de spécialistes. La différence s'estompe donc entre scènes de cour et de ville, les scènes "pauvres " utilisant les mêmes feintes.

4 Si l'on peut donc mieux repérer la porosité de ces milieux et la manière dont se forgent des savoir-faire communs et des importations de procédés, la circulation de ces savoirs, réduits en art dans les traités techniques, et leur adaptation dans la pratique, reste néanmoins posée. Le numéro permet ainsi de mieux tracer ce qui relève de l'héritage des savoirs antiques et médiévaux : à partir des textes disponibles, de ceux de Villars de Honnecourt au Disdacalion d'Hugues de Saint-Victor - qui place le théâtre parmi les arts mécaniques - c'est dans la longue histoire des arts mécaniques que les questions de techniques théâtrales peuvent être resituées dans leur faculté à combiner les nouveautés techniques du Quattrocento, comme le rappelle Pascal Brioist à propos de Léonard de Vinci (p. 49). Ces dispositifs déploient une relation forte à l'exploration de la nature des mécaniques où s'opère la circulation entre les savoirs d'ingénierie et des questions complexes de conception. Pour autant leur réappropriation demeure l'objet d'hypothèses. Ainsi, le traité de Vitruve, De architectura et les écrits de Héron d'Alexandrie semblent avoir été déterminants. Si l'influence de ce dernier semble bien établie en Italie, l'édition en latin décrivant machines automates, horloges à eau, à vapeur, et fontaines, date de 1575 tandis que les Pneumatica, Libro degli artifizii spititali ne sont traduits en italien qu'en 1582 par l'ingénieur Vannochio Biringuccio (qui les utilise dans une fête pour le prince Gonzague de Mantoue). Mais ce traité semble avoir été connu et utilisé bien avant son impression : la présence des textes alexandrins, voire d'une traduction arabe, est probable et les rend mobilisables, y compris par Leonard dès la fin du $\mathrm{XV}^{\mathrm{e}}$ siècle. Les Pneumatica qui connaissent une série d'éditions et rééditions successives entre 1575 et 1647 sont un texte bien connu à Florence, Mantoue, Parme, Sienne, Urbino et qui, sans être traduit en français, voyage via des artistes, lettrés et 
scénographes italiens en France et aux Pays-Bas dès la fin du xvI $\mathrm{e}^{\mathrm{e}}$ siècle (notamment via les troupes italiennes venues à Paris). La machine y est l'incarnation de l'ingéniosité et de l'artifice, elle crée son "propre mouvement " comme l'indique encore Furetière. Vases communicants, compression-dépression de l'air, flux de liquide et d'air, mise en mouvement des mécanismes (p.63), la circulation de ces connaissances est prédominante dans le contexte des spectacles curiaux - à l'instar des machines conçues pour un ballet allégorico-mécanique par Guillaume de Colleter, où le régisseurmachiniste, crée une "feinte machinée " pour la cour de Lorraine au début du XVII ${ }^{e}$ siècle - et les savoirs des scénographes italiens se diffusent dans l'ensemble des scènes européennes, une influence qui se prolonge au cours du XVII siècle via la comédie italienne. Ainsi, le théâtre italien de Gherardi, édite nombre de gravures et dessins. Cette mise en art des savoirs scénographiques fait des émules, comme les décors imaginés par Jean Bérain père et fils; ils constituent un corpus qui inspire encore les conceptions de la machinerie de l'opéra (trappes, cintres, poulies \& contrepoids, etc.), dessinées et expliquées en 1770 par l'architecte expert LouisFrançois Petit Radel, pour la cinquantaine de planches de Machines de théâtre parues dans l'Encyclopédie en 1772.

5 Un dernier aspect permet de questionner sur la longue durée l'évolution de la perception des machineries et des effets spéciaux, relativement aux attentes des spectateurs et au sens donné à la représentation. On assiste progressivement à une forme de déplacement qui change la perception des effets. La mimesis n'est guère l'effet recherché initialement: les moyens techniques ne sont pas cachés, voire ils sont exemplifiés. Ainsi dans le cas du Mystère des Actes des Apôtres de Bourges en 1536, "l'outrance des effets doit fournir le merveilleux »; il ne s'agit là ni d'illusion, ni d'imitation (p. 21). Sur la scène florentine $\mathrm{du} \mathrm{xv}^{\mathrm{e}}$ siècle, le développement de machines scéniques complexes fournissant leurs lots d'anges, de nuées et d'effets sonores et lumineux fait du décor une métonymie du spectacle d'ascension. Pour les fêtes de quartiers célébrant les patrons de cités à Florence, les machines ascensionnelles sont une représentation de l'ineffable (p.33) et du paradis, susceptible d'impressionner les fidèles et de créer un imaginaire de l'au-delà. Le théâtre baroque en France n'est pas lui non plus dépourvu d'« effets » ni d'appareil, ni même le théâtre élisabéthain qui de fait n'a rien d'une scène nue. L'art métonymique de l'accessoire y prévaut et il s'agit de créer sans cesse la surprise - dans un contexte de concurrence entre les théâtres - afin d'«impressionner et charmer les spectateurs» par des nouveautés techniques ingénieuses auxquelles les spectateurs prennent plaisir. L'action dramatique que les publics perçoivent avec les moyens techniques ne se fonde pas sur le registre de l'illusion. Une véritable poétique de la machine se prolonge y compris au travers de l'émergence de nouveaux genres en France à la fin du XvII siècle avec le ballet, et dont le principe repose sur des intrigues indépendantes et des changements à vue rythmés par l'introduction de décors nouveaux. Aux palais, jardins, temples, villes mythologiques héritées du genre sérieux de l'opéra, l'appareil scénique du ballet à l'Académie royale de musique préfère bientôt des présentations plus ancrées dans le monde contemporain: paysages de France (fleuve, scène champêtre, foire contemporaine) et goût du voyage (fêtes vénitiennes).

6 La machine de théâtre a cependant connu des mutations interprétatives. Chez Vinci, les mécanismes mathématiques proposent une métaphore de l'univers créé par Dieu «décryptant les secrets du monde» (p.56) pour les spectateurs. Cette dimension 
démiurgique s'incarne dans des automates, du lion automate au colosse de la musique imaginée par Morel. Dans l'économie du spectacle princier se met en place un système apologétique et allégorique, où les éléments visuels et textuels sont porteurs de sens. La machine s'inscrit alors dans la sphère morale, celle de l'artifice et de la "machination ", elle sert des représentations savantes et des enjeux politiques afin de questionner les illusions comme métaphore du monde ainsi que Molière le met en scène dans Les amants magnifiques par la présence sur scène du machiniste-scénographe qui dévoile les rouages cachés et révèle l'illusion; cette dimension morale perdure dans les contributions que Louis de Cahusac écrit pour l'Encyclopédie au milieu du XVIII ${ }^{e}$ siècle, décrivant les machines d'opéra avec l'ambition d'unifier une conception générale, matérielle et poétique de la représentation spectaculaire. En phase avec l'esprit encyclopédique, il relie science, arts et techniques, tandis qu'au même moment le discrédit touche ces spectacles de l'enchantement, dénigrés pour la médiocrité de leurs trucages. L'évolution des machines et des aspects scéniques au XVIII siècle se distancie donc du théâtre du merveilleux et des machines héritées du théâtre baroque prônant une approche plus naturelle et moins portée sur les effets.

7 On peut remarquer que ce goût pour l'enchantement semble néanmoins perdurer dans d'autres sphères que celle de la représentation théâtrale, dans les spectacles techniques, expériences publiques, promenades, ateliers et boutiques des artisans inventeurs, qui n'ont de cesse de mettre en scène la performativité des accomplissements techniques dans une tension constante entre fascination émerveillée et compréhension des procédés ${ }^{2}$. Le dossier éclaire donc un domaine essentiel de la culture technique. Mais loin de se limiter aux scènes de théâtre, le sujet est à verser dans une réflexion plus globale. Il met en lumière la place des machineries et des feintes dans l'évolution du goût de publics nombreux qui expérimentent leur rapport au savoir-faire, aux effets merveilleux et à l'artifice ${ }^{3}$. On pourrait réinsérer les mutations du sens donné aux machines dans des dynamiques plus larges, afin d'en étudier les migrations et les persistances selon les audiences et les lieux, y compris dans les transformations du jeu métaphorique qui connaît de nouvelles appropriations morales et matérielles au tournant du XVIII ${ }^{e}$ et $\mathrm{XIX}^{\mathrm{e}}$ siècles.

\section{BIBLIOGRAPHIE}

HILAIRE-PÉREZ Liliane, « Les boutiques d'inventeurs à Londres et à Paris au XVIII siècle : jeux de l'enchantement et de la raison citoyenne ", dans COQUERY Natacha (dir.), La boutique et la ville, commerces, commerçants, espaces et clientèles, CEHVI, Tours, 2000, p. 171-218.

TKACZYK Viktoria, «L'École d'ingénierie scénique de Giulio Parigi (1608-1680) », Artefact.

Techniques, histoire et sciences humaines, $\mathrm{n}^{\circ}$ 4, 2016, p. 99-117. 


\section{NOTES}

1. On pourra consulter à ce sujet Tkaczyk, 2016.

2. Hilaire-Pérez, 2000.

3. Effets spéciaux et artifices, sous la direction d'Isabelle Balsamo, Terrain, anthropologie et sciences humaines, $\mathrm{n}^{\circ}$ 46, mars 2006.

\section{AUTEURS}

\section{MARIE THÉBAUD-SORGER}

CNRS, Associate research professor Maison Française d'Oxford 